\title{
First-principles based multiscale model of piezoelectric nanowires with surface effects
}

\author{
M.-T.Hoang, J.Yvonnet, ${ }^{*}$ A. Mitrushchenkov, and G. Chambaud \\ Université Paris-Est, MSME UMR 8208 CNRS, \\ 5 Bd Descartes 77454 Marne-la-Vallée CEDEX 2, France
}

(Dated: December 2, 2012)

\begin{abstract}
A continuum model of nanowires incorporating surface piezoelectricity is proposed which extends the electric enthalpy energy with surface terms. The corresponding equations are solved by a numerical method using finite elements technique. A methodology is introduced to compute the surface piezoelectric coefficients by first-principles calculations through the Berry phase theory. We provide the $e_{33}^{s}, e_{31}^{s}$ and $e_{15}^{s}$ piezoelectric coefficients of $(10 \overline{1} 0)$ surfaces for hexagonal wurtzite nanowires made of $\mathrm{GaN}, \mathrm{ZnO}$ and $\mathrm{AlN}$. The effective piezoelectric coefficient along the axis of the nanowire is found to increase when the diameter decreases, for the three studied materials. Finally, the solution of the continuum model is compared with large-size first-principles calculations on piezoelectric nanowires.
\end{abstract}

PACS numbers: Valid PACS appear here

\section{INTRODUCTION}

Recent experimental studies have evidenced higher performances of piezoelectric nanostructures as compared to bulk materials. ${ }^{1-10}$ Very recently, Qi and M. C. McAlpine have developed Pb-Zr-Ti (PZT) ceramics nanowires on a polymer and have reported the highest piezoelectric performance. ${ }^{11}$ These structures have then attracted a tremendous attention due to potential applications in energy harvesting, ${ }^{12-16}$ sensors,${ }^{17}$ piezoelectric output voltage scale ${ }^{18}$ and self-powered nanodevices, ${ }^{2}$ among many others.

Piezoelectric coefficients $e_{33}$ and $e_{31}$ of nanowires can be measured by PFM (Piezoresponse Force Microscopy) and AFM tip ${ }^{1-4}$ (Atomic Force Microscope). Espinosa et al. ${ }^{9,10}$ showed that a giant piezoelectric constant is observed in GaN and $\mathrm{ZnO}$ nanowires with very small diameters (about $2.5 \mathrm{~nm}$ for GaN nanowires). Another important aspect is the wide band gap of semi-conducting nanowires, which is influenced by surface effects for small diameters. ${ }^{19,20}$

In addition, numerical investigations involving firstprinciples calculations confirm these effects. ${ }^{19,21-26}$ Following $\mathrm{Li}$ et al., ${ }^{27}$ Dai et al. ${ }^{28}$ evaluated the effects of surface piezoelectricity on $\mathrm{ZnO}, \mathrm{SrTiO}_{3}$ and $\mathrm{BaTiO}_{3}$ systems by using first-principles and core-shell interatomic simulations.

In this paper, we propose a continuum model of piezoelectric nanowires with surface effects. We extend to piezoelectricity an approach previously proposed ${ }^{29-31}$ for modeling mechanical surface effects in nanowires. A procedure based on first-principles calculations is developed to compute the surface piezoelectric coefficients using slab surface models. A finite element methodology is used to solve the equations of the continuum model for a geometry involving a hexagonal section, corresponding to wurtzite monocrystal nanowires made of GaN, AlN and $\mathrm{ZnO}$. We then compare the values of the effective $e_{33}$ coefficient obtained by our model with full first-principles calculations on several nanowires with different diameters to assess the accuracy of the model.

\section{CONTINUUM MODEL FOR SURFACE PIEZOELECTRICITY}

Piezoelectricity is due to the atomic scale polarization (see a review of electromechanical phenomena in semiconductor nanostructures in reference). ${ }^{32}$ It is caused by the non centrosymmetric crystal structure of certain materials, which results in an effective change of polarization in response to an applied mechanical strain.

When considering surface effects in nanostructures, the total energy (enthalpy) of the system can be written as bulk and surface contributions:

$$
W=\int_{V} \omega^{b}\left(\varepsilon_{i j}, E_{i}\right) d V+\int_{S} \omega^{s}\left(\varepsilon_{i j}^{s}, E_{i}^{s}\right) d S
$$

where $\omega^{b}$ and $\omega^{s}$ are the bulk enthalpy density and surface enthalpy density, respectively, while $\varepsilon$ and $E_{i}$ denote the strain second order tensor and the electric field vector. We assume the following forms for the energy (enthalpy) density functions:

$$
\omega^{b}\left(\varepsilon_{i j}, E_{i}\right)=\frac{1}{2} C_{i j k l} \varepsilon_{i j} \varepsilon_{k l}-e_{i j k} E_{i} \varepsilon_{j k}-\frac{1}{2} \alpha_{i j} E_{i} E_{j}
$$

and

$$
\begin{gathered}
\omega^{s}\left(\varepsilon_{i j}^{s}, E_{i}^{s}\right)=\frac{1}{2} C_{i j k l}^{s} \varepsilon_{i j}^{s} \varepsilon_{k l}^{s}-e_{i j k}^{s} E_{i}^{s} \varepsilon_{j k}^{s}-\frac{1}{2} \alpha_{i j}^{s} E_{i}^{s} E_{j}^{s} \\
+\tau_{i j}^{s} \varepsilon_{i j}^{s}
\end{gathered}
$$

where $C_{i j k l}, e_{i j k}, \alpha_{i j}$ are the bulk elastic fourth-order tensor, the bulk piezoelectric third-order tensor and the bulk second-order dielectric tensor, respectively, while $C_{i j k l}^{s}, e_{i j k}^{s}, \alpha_{i j}^{s}$ are their surface counterparts and $\tau_{i j}^{s}$ is the surface residual stress. These tensors have the following symmetries 


$$
\begin{gathered}
C_{i j k l}=C_{k l i j}=C_{i j l k}=C_{j i k l}, \\
e_{k i j}=e_{k j i} ; \quad \alpha_{i k}=\alpha_{k i} .
\end{gathered}
$$

In Eq. (3), $\varepsilon^{s}$ and $E^{s}$ are the surface strain tensor and surface electric field vector. In this work, the surface vectors $v^{s}$ and second-order tensors $A_{i j}^{s}$ are defined from their bulk definition through the use of an orthogonal projector operator $T_{i j}=\delta_{i j}-n_{i} n_{j}$, describing the projection on the plane tangent to the surface $S$ at $x \in S$, where $n_{i}$ is the unit vector normal to $S$ at $x$. Then, we have $v^{s}=T_{i j} v_{j}$ and $A_{i j}^{s}=T_{i k} A_{k l} T_{l j}$. The constitutive equations for bulk and surface can be established from (2)-(3) as

$$
\begin{gathered}
\sigma_{i j}=\frac{\partial \omega^{b}}{\partial \varepsilon_{i j}}=C_{i j k l} \varepsilon_{k l}-e_{k i j} E_{k}, \\
D_{i}=-\frac{\partial \omega^{b}}{\partial E_{i}}=e_{i k l} \varepsilon_{k l}+\alpha_{i k} E_{k}, \\
\sigma_{i j}^{s}=\frac{\partial \omega^{s}}{\partial \varepsilon_{i j}^{s}}=C_{i j k l} \varepsilon_{k l}^{s}-e_{k i j}^{s} E_{k}^{s}+\tau_{i j}^{s},
\end{gathered}
$$

and

$$
D_{i}^{s}=-\frac{\partial \omega^{s}}{\partial E_{i}^{s}}=e_{i k l}^{s} \varepsilon_{k l}^{s}+\alpha_{i k}^{s} E_{k}^{s}
$$

where $\sigma$ is the stress tensor and $D$ the electric displacement. The polarization vector for bulk and surface, denoted by $P$ and $P^{s}$ respectively, are related to the electric displacement vectors by

$$
D_{i}=P_{i}+\epsilon_{0} E_{i}, \quad D_{i}^{s}=P_{i}^{s}+\epsilon_{0} E_{i}^{s},
$$

where $\epsilon_{0}$ is the permittivity of vacuum. We can finally express the bulk and surface piezoelectric coefficients as

$$
e_{i j k}=\frac{\partial D_{i}}{\partial \varepsilon_{j k}}=\frac{\partial P_{i}}{\partial \varepsilon_{j k}}, \quad e_{i j k}^{s}=\frac{\partial D_{i}^{s}}{\partial \varepsilon_{j k}^{s}}=\frac{\partial P_{i}^{s}}{\partial \varepsilon_{j k}^{s}} .
$$

The effective polarization can be defined as

$$
P_{i}^{e f f}=\frac{1}{V} \frac{\partial W}{\partial E_{i}^{e f f}}
$$

where $E_{i}^{e f f}$ denotes an effective electric field such that

$$
E_{i}^{e f f}=\frac{1}{V} \int_{V} E_{i} d V
$$

Then we have, using (1):

$$
\begin{gathered}
P_{i}^{e f f}=\frac{1}{V} \int_{V} \frac{\partial \omega^{b}}{\partial E_{i}^{e f f}} d V+\frac{1}{V} \int_{S} \frac{\partial \omega^{s}}{\partial E_{i}^{e f f}} d S \\
=\frac{1}{V} \int_{V} \frac{\partial \omega^{b}}{\partial E_{j}} \frac{\partial E_{j}}{\partial E_{i}^{e f f}} d V+\frac{1}{V} \int_{S} \frac{\partial \omega^{s}}{\partial E_{j}^{s}} \frac{\partial E_{j}^{s}}{\partial E_{i}^{e f f}} d S .
\end{gathered}
$$

From (13) we obtain

$$
\frac{\partial E_{i}^{e f f}}{\partial E_{j}}=\frac{1}{V} \int_{V} \delta_{i j} d V=\delta_{i j}
$$

Furthermore, by noting that $E_{i}^{s}=T_{i j} E_{j}$, it yields

$$
\frac{\partial E_{i}^{s}}{\partial E_{j}^{e f f}}=T_{i k} \frac{\partial E_{k}}{\partial E_{j}^{e f f}}=T_{i k} \delta_{k j}=T_{i j}
$$

and

$$
\frac{\partial \omega^{s}}{\partial E_{j}^{s}} \frac{\partial E_{j}^{s}}{\partial E_{i}^{e f f}}=P_{j}^{s} T_{i j}=P_{i}^{s} .
$$

Thus, we finally obtain

$$
P_{i}^{e f f}=\frac{1}{V} \int_{V} P_{i} d V+\frac{1}{V} \int_{S} P_{i}^{s} d S .
$$

The domain is subjected to Dirichlet boundary conditions, i.e. displacements and potential are prescribed over respective portions of the external boundary of the domain. To solve the problem, the total energy (1) of the system submitted to an applied external force $\bar{F}_{i}$ and external electric displacement $\bar{D}_{i}$, is expressed, in the absence of body forces by

$$
W-\int_{S_{F}} \bar{F}_{i} u_{i} d S-\int_{S_{D}} \bar{D}_{i} n_{i} \phi d S
$$

where $S_{F}$ and $S_{D}$ denote respectively the traction and electric displacement surfaces and $\phi$ is the electric potential. The total energy expressed in (20) is optimized with respect to the nodal displacements and nodal electric potentials associated to a finite element mesh discretizing the domain $\Omega$.

\section{FIRST-PRINCIPLES PIEZOELECTRIC CALCULATIONS}

The calculations of the piezoelectric coefficients in the framework of periodic Density Functional Theory (DFT) were done using the Berry phase approach. ${ }^{33,34}$ In this 
method, the piezoelectric coefficients $e_{i k}$ are obtained as $^{35}$

$$
e_{i k}=-\frac{1}{2 \pi V} \sum_{\alpha} \frac{\partial \phi_{\alpha}}{\partial \varepsilon_{k}} a_{\alpha i}
$$

where $V$ is the unit cell volume, the sum runs over unit cell directions, $\mathbf{a}_{\alpha}$ being the corresponding unit cell vectors, and $\phi_{\alpha}$ the Berry phase in the direction $\alpha$, which is a sum of nuclear and electronic components,

$$
\phi_{\alpha}=\phi_{\alpha}^{n u c}+\phi_{\alpha}^{e l e c}
$$

with nuclear part as a simple geometric sum

$$
\phi_{\alpha}^{n u c}=\sum_{i A} B_{\alpha i} R_{A i} Z_{A},
$$

with the sum running over atoms in the unit cell $A$ and Cartesian components $i, B_{\alpha i}$ being reciprocal unit cell vectors, $R_{A i}$ and $Z_{A}$ the coordinates and charges of the nuclei, respectively. The electronic part is evaluated by integrating over the Brillouin zone,

$$
\phi_{\alpha}^{e l e c}=\frac{1}{\Omega} \sum_{n} \int_{B Z}\left\langle u_{n \mathbf{k}}\left|-i \mathbf{B}_{\alpha} \nabla_{\mathbf{k}}\right| u_{n \mathbf{k}}\right\rangle d \mathbf{k},
$$

where $\Omega=(2 \pi)^{3} / V$ is the Brillouin zone volume and the sum runs over all occupied orbitals in DFT wave function with $u_{n \mathbf{k}}$ being the corresponding Bloch functions.

These total (nuclear and electronic) Berry phases are calculated by CRYSTAL09 code for a set of deformations (we used a total of 11 calculations for each distortion with the deformation parameter $\varepsilon$ running from -0.005 to 0.005 with a step of 0.001 ) and then the derivative of the Berry phase with respect to $\varepsilon$ is calculated numerically (actually, we have fitted $\phi$ as a third order polynomials of $\varepsilon)$. This allows calculating the piezoelectric parameters for bulk (3D), slabs (2D) and nanowires (1D) using the same approach.

\section{BULK CALCULATIONS}

The three semiconductor systems, GaN, ZnO and AlN, studied here, have wurtzite structure and are tetrahedrally coordinated. This structure has a hexagonal Bravais lattice with four atoms per unit cell (see Fig. 1). The geometry is fully defined by the length of hexagonal edge $a$, the height $c$ of the prism, and by the relative distance $u$ between the two atoms of different type.

For a wurtzite crystal, the piezoelectric tensor has three independent components in Voigt's notation $e_{33}$, $e_{31}$ and $e_{15}=e_{24}$ : the first two components $e_{33}, e_{31}$ measure the variation of polarization along the axis 3 by a uniform strain in the plane normal to the 3-direction. The third constant $e_{15}$ describes the polarization induced

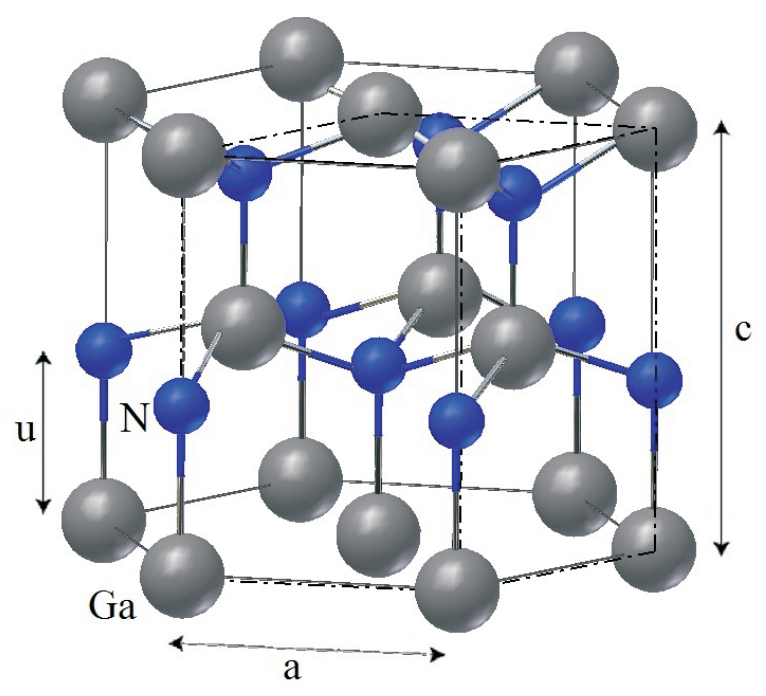

FIG. 1: Wurtzite structure and unit cell (dashed lines) for $\mathrm{GaN}$

by a shear strain perpendicularly to the axis 3 . The matrix of bulk piezoelectric coefficients is then defined as follows

$$
e=\left[\begin{array}{cccccc}
0 & 0 & 0 & 0 & e_{15} & 0 \\
0 & 0 & 0 & e_{15} & 0 & 0 \\
e_{31} & e_{31} & e_{33} & 0 & 0 & 0
\end{array}\right]
$$

The strain tensor is characterized in Voigt's notation by the components $\varepsilon=\left[\varepsilon_{1}, \varepsilon_{2}, \varepsilon_{3}, \varepsilon_{4}, \varepsilon_{5}, \varepsilon_{6}\right]$. For wurtzite, the bulk elastic tensor can be expressed by five independent constants: $C_{11}, C_{12}, C_{13}, C_{33}$, and $C_{44}$ (see more details in). ${ }^{29-31}$ Bulk elastic parameters have been characterized in many other studies, including our previous papers. ${ }^{29,31}$ In this work, we have also computed the bulk piezoelectric coefficients for $\mathrm{GaN}, \mathrm{ZnO}$, and $\mathrm{AlN}$ by first-principles calculations in Table I for comparison with other studies. The bulk piezoelectric constants are given by:

$$
e_{i k}^{a b}=\frac{1}{V_{\text {cell }}} \frac{\partial P_{i}^{a b}}{\partial \varepsilon_{k}}, \quad i=1,2,3 ; k=1,2, \ldots, 6
$$

where $P_{i}^{a b}$ is the total polarization per unit cell computed by first-principles calculations and $V_{\text {cell }}$ is the unit cell volume defined as:

$$
V_{\text {cell }}=a^{2} c \frac{\sqrt{3}}{2} .
$$

The elastic constants have been evaluated at the atomistic level by first-principles calculations, through the CRYSTAL code,${ }^{36}$ using the PBESOL and PWGGA DFT functionals. For GaN and AlN, the calculations with hybrid PBE0 were also performed. For bulk materials, we also give the B3LYP results. In the present work, 
TABLE I: Bulk geometric (in $\AA$ ) and elastic parameters (in $\mathrm{GPa})$ for wurtzite $\mathrm{GaN}, \mathrm{ZnO}$ and $\mathrm{AlN}$.

\begin{tabular}{|c|c|c|c|c|c|c|c|}
\hline Method & $a$ & $c$ & $C_{11}$ & $C_{33}$ & $C_{13}$ & $C_{12}$ & $C_{55}$ \\
\hline \multicolumn{8}{|l|}{$G a N$} \\
\hline PWGGA & 3.200 & 5.207 & 361.1 & 397.2 & 94.6 & 130.6 & 93.3 \\
\hline PBESOL & 3.171 & 5.160 & 378.0 & 419.4 & 107.1 & 144.3 & 94.2 \\
\hline PBE0 & 3.178 & 5.158 & 396.6 & 432.3 & 108.0 & 146.4 & 103.8 \\
\hline B3LYP & 3.206 & 5.211 & 381.3 & 413.3 & 91.6 & 129.3 & 104.2 \\
\hline Expt. ${ }^{41}$ & & & 361.2 & 390.3 & 93.1 & 130.9 & 98.0 \\
\hline \multicolumn{8}{|l|}{$\mathrm{ZnO}$} \\
\hline PWGGA & 3.276 & 5.278 & 203.3 & 215.1 & 103.3 & 117.8 & 34.0 \\
\hline PBESOL & 3.237 & 5.220 & 215.9 & 229.6 & 119.2 & 132.3 & 32.0 \\
\hline $\mathrm{PBE0}^{30}$ & 3.195 & 5.165 & 224.9 & 219.3 & 112.4 & 128.5 & 41.3 \\
\hline $\mathrm{B}^{2} \mathrm{LYP}^{30}$ & 3.261 & 5.216 & 217.2 & 229.0 & 98.8 & 116.1 & 43.1 \\
\hline $\mathrm{HF}^{45}$ & & & 246.2 & 241.3 & 104.3 & 127.5 & 56.4 \\
\hline Expt. ${ }^{43}$ & 3.247 & 5.203 & & & & & \\
\hline Expt. ${ }^{44}$ & & & 206 & 211 & 118 & 117 & 44.3 \\
\hline \multicolumn{8}{|l|}{$A l N$} \\
\hline PWGGA & 3.125 & 5.008 & 393.5 & 377.9 & 104.6 & 136.3 & 116.4 \\
\hline PBESOL & 3.111 & 4.980 & 397.0 & 376.9 & 114.2 & 145.6 & 114.4 \\
\hline PBE0 & 3.105 & 4.966 & 424.0 & 401.9 & 115.0 & 148.6 & 127.0 \\
\hline B3LYP & 3.118 & 5.003 & 420.9 & 407.3 & 99.3 & 134.3 & 129.8 \\
\hline $\mathrm{HF}^{47}$ & 3.117 & 4.982 & 464 & 409 & 116 & $\begin{array}{c}14.0 \\
149\end{array}$ & 128 \\
\hline Expt. ${ }^{48}$ & 3.1111 & 4.9788 & & & & & \\
\hline Expt. ${ }^{46}$ & & & 410.5 & 388.5 & 98.9 & 148.5 & 124.6 \\
\hline
\end{tabular}

the following Gaussian basis sets have been employed with CRYSTAL: $86-411 \mathrm{~d} 31 \mathrm{G}$ for $\mathrm{Zn},{ }^{36}$ 86-21G for $\mathrm{Al},{ }^{37}$ 86-4111d41G for Ga, ${ }^{38,39} 8-411 \mathrm{G}$ for O and $6-31 \mathrm{~d} 1 \mathrm{G}$ for $\mathrm{N} .{ }^{40}$ Note that the total polarization is calculated using DFT method described above for several values of strains $\varepsilon_{k}$. The derivatives have been evaluated by a polynomial fitting.

For comparison purposes, we present complete results for all three species in Table II, together with previously calculated values and experimental results when available. Nakamura et al. ${ }^{41}$ provided experimental results which differ strongly from the other available values reported in Table II. A discussion on this discrepancy is provided by the author in the mentioned paper.

The standard units for piezoelectric constants (pervolume) are $\mathrm{C} / \mathrm{m}^{2}$. To convert these to per-atom units of $10^{-16} \mu \mathrm{C} \times \AA /$ atom, ${ }^{21}$ the following relationship is used:

$$
e_{i k}\left(\mathrm{C} / \mathrm{m}^{2}\right)=e_{i k}\left(10^{-16} \mu \mathrm{C} \times \AA / \text { atom }\right) \mathrm{N}_{\text {atoms }} / \mathrm{V}_{\text {cell }} .
$$

TABLE II: Bulk piezoelectric parameters (in $\mathrm{C} / \mathrm{m}^{2}$ ) for wurtzite $\mathrm{GaN}, \mathrm{ZnO}$ and $\mathrm{AlN}$.

\begin{tabular}{|c|c|c|c|}
\hline Method & $e_{33}$ & $e_{31}$ & $e_{15}$ \\
\hline \multicolumn{4}{|l|}{$G a N$} \\
\hline PWGGA & 0.769 & -0.377 & -0.314 \\
\hline PBESOL & 0.904 & -0.520 & -0.373 \\
\hline PBE0 & 0.944 & -0.513 & -0.378 \\
\hline $\mathrm{LDA}^{26}$ & 0.73 & -0.49 & \\
\hline $\mathrm{LDA}^{25}$ & 0.63 & -0.32 & \\
\hline Expt. ${ }^{49}$ & 1 & -0.36 & -0.3 \\
\hline Expt. ${ }^{41}$ & 0.28 & 0.07 & 0.12 \\
\hline \multicolumn{4}{|l|}{$\mathrm{ZnO}$} \\
\hline PWGGA & 1.276 & -0.561 & -0.535 \\
\hline PBESOL & 1.389 & -0.706 & -0.591 \\
\hline $\mathrm{LDA}^{26}$ & 0.89 & -0.51 & \\
\hline $\mathrm{HF}^{22}$ & 1.19 & -0.55 & -0.46 \\
\hline $\mathrm{DFT}^{28}$ & 1.22 & -0.59 & \\
\hline Expt. ${ }^{49}$ & 1.55 & -0.58 & -0.48 \\
\hline \multicolumn{4}{|l|}{$A l N$} \\
\hline PWGGA & 1.489 & -0.551 & -0.336 \\
\hline PBESOL & 1.647 & -0.618 & -0.374 \\
\hline PBE0 & 1.598 & -0.570 & -0.369 \\
\hline B3LYP & 1.341 & -0.457 & -0.309 \\
\hline $\mathrm{LDA}^{26}$ & 1.46 & -0.60 & \\
\hline $\mathrm{LDA}^{25}$ & 1.29 & -0.38 & \\
\hline Expt. ${ }^{49}$ & 1.32 & -0.57 & -0.48 \\
\hline
\end{tabular}

\section{SURFACE PIEZOELECTRIC PROPERTIES PARAMETERS CALCULATIONS}

For wurtzite, the full matrix of surface piezoelectric constants for $\mathrm{GaN}, \mathrm{ZnO}$ and $\mathrm{AlN}$ nanowire is defined as

$$
e^{s}=\left[\begin{array}{ccc}
0 & 0 & e_{15}^{s} \\
e_{31}^{s} & e_{33}^{s} & 0
\end{array}\right]
$$

We have proposed in our previous papers ${ }^{29-31}$ a methodology to identify surface elastic parameters associated to an infinitely thin surface from atomistic slab models. In this work, we extend this procedure to compute the surface piezoelectric parameters. As the isolated surface does not exist in nature, we have to proceed via a series of slab calculations with $(10 \overline{1} 0)$ free surfaces, varying the number of atomic layers $(n)$ in the slab, see Figure 2. The number of atoms in the slab unit cell is $N=4 n$. To avoid the ambiguity of defining slab and surface widths, and assuming that surface corresponds to 1 atomic layer (including atoms of both types), we plot the per-atom properties (energy of polarization) as a function of surface atomic weight, $w=\frac{2}{n}$ where factor 2 comes from free surfaces on both sides of the slab. Assuming additive model, the dependence should be linear, e.g.

$$
P_{i}^{s l a b}(w)=w P_{i}^{\text {surf }}+(1-w) P_{i}^{\text {slab }}(w \rightarrow 0),
$$




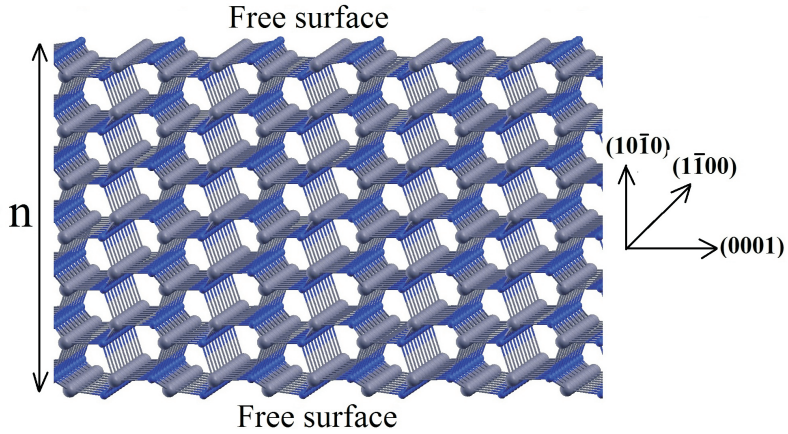

FIG. 2: Slab system of a wurtzite structure with (10̄0) surface and $n=7$ layers.

leading to a similar relation for slab per-atom piezoelectric coefficient

$$
e_{i j}^{a, s l a b}(w)=w e_{i j}^{a, s u r f}+(1-w) e_{i j}^{a, s l a b}(w \rightarrow 0) .
$$

The limiting values $e_{i j}^{a, s l a b}(w \rightarrow 0)$ are related to bulk parameters as:

$$
\begin{aligned}
& e_{33}^{a, \text { slab }}(w \rightarrow 0)=e_{33}^{a, \text { bulk }}-\frac{C_{13}^{\text {bulk }}}{C_{11}^{\text {bulk }} e_{31}^{a, \text { bulk }}} \\
& e_{31}^{a, \text { slab }}(w \rightarrow 0)=e_{31}^{a, \text { bulk }} \frac{C_{11}^{\text {bulk }}-C_{12}^{\text {bulk }}}{C_{11}^{\text {bulk }}}
\end{aligned}
$$

and $e_{15}^{a, s l a b}(w \rightarrow 0)=e_{15}^{a, b u l k}$.

Thus, by fitting $e_{i j}^{a, s l a b}(w)$ to a linear function, we can obtain the surface per-atom piezoelectric coefficients which can be further converted to per-surface units, to be used in continuous model, via

$$
e_{i k}^{s}(\mathrm{nC} / \mathrm{m})=\frac{4}{a c} e_{i k}^{s}\left(10^{-32} \mathrm{Cm} / \text { atom }\right) .
$$

using the fact that surface unit cell contains 4 atoms and has an area of $S=a c$ with $a$ and $c$ being bulk unit cell parameters.

In figures 3,4 and 5 , we show the plots of the $e_{33}, e_{31}$ and $e_{15}$ coefficients as a function of $w$, for $\mathrm{GaN}, \mathrm{ZnO}$ and AlN materials. We note that the linear model is generally very good with one exception of $e_{33}$ coefficient for $\mathrm{ZnO}$. For the latter case, the coefficient $e_{33}$ varies very little with slab size and stays close to bulk value. This, most probably, indicates that surface and bulk parameters are practically identical and the variation is caused by numerical errors.

Finally, the computed values of the surface coefficients, using different DFT functionals, are summarized in Table IV. Note that the values differ significantly from the results obtained by Li et al. ${ }^{27}$ and Dai et al. ${ }^{28}$ The main reason is the difference between the studied systems. In the mentioned papers the free surfaces considered in the slab
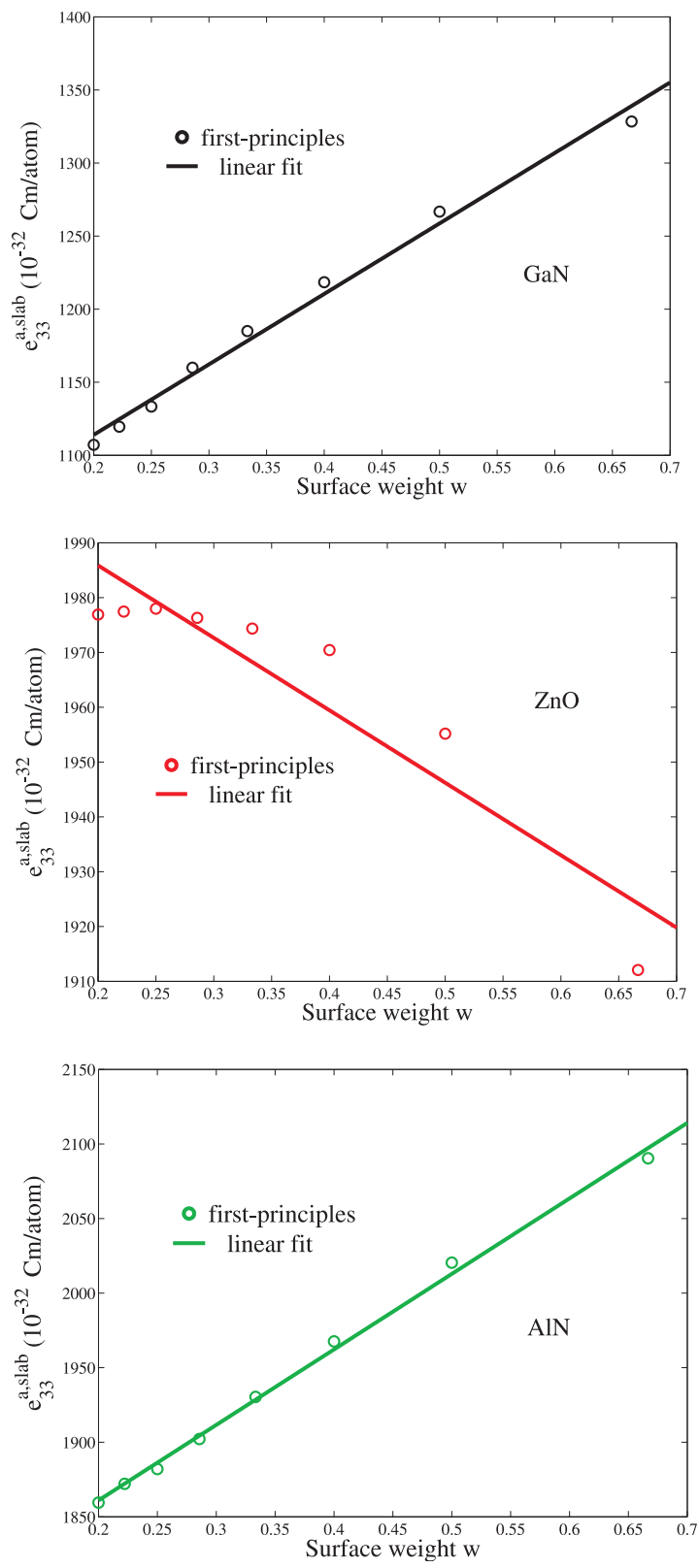

FIG. 3: Plots of $e_{33}^{a, s l a b}$ (in $10^{-32} \mathrm{Cm} /$ atom) as a function of surface weight $w$, used for computing the surface piezoelectric parameter $e_{33}^{s}$ for $\mathrm{GaN}, \mathrm{ZnO}$ and $\mathrm{AlN}$.

model were (0001) surfaces and the sample was stretched in the direction normal to this surface.

Elastic surface properties for $\mathrm{ZnO}$ and AlN have been computed in our previous papers. ${ }^{29,31}$ In this work, we also calculated elastic surface constants for GaN. For wurtzite, there are four elastic surface constants $C_{11}^{s}, C_{13}^{s}, C_{33}^{s}$, and $C_{55}^{s}$ and two residual stress components $\tau_{1}^{s}$, $\tau_{3}^{s}$ (see Table III). Conversion from Hartree/atom to $\mathrm{N} / \mathrm{m}$ is done through $\mathrm{C}(\mathrm{N} / \mathrm{m})=\frac{4}{S} \mathrm{C}($ Hartree/atom $)$. 

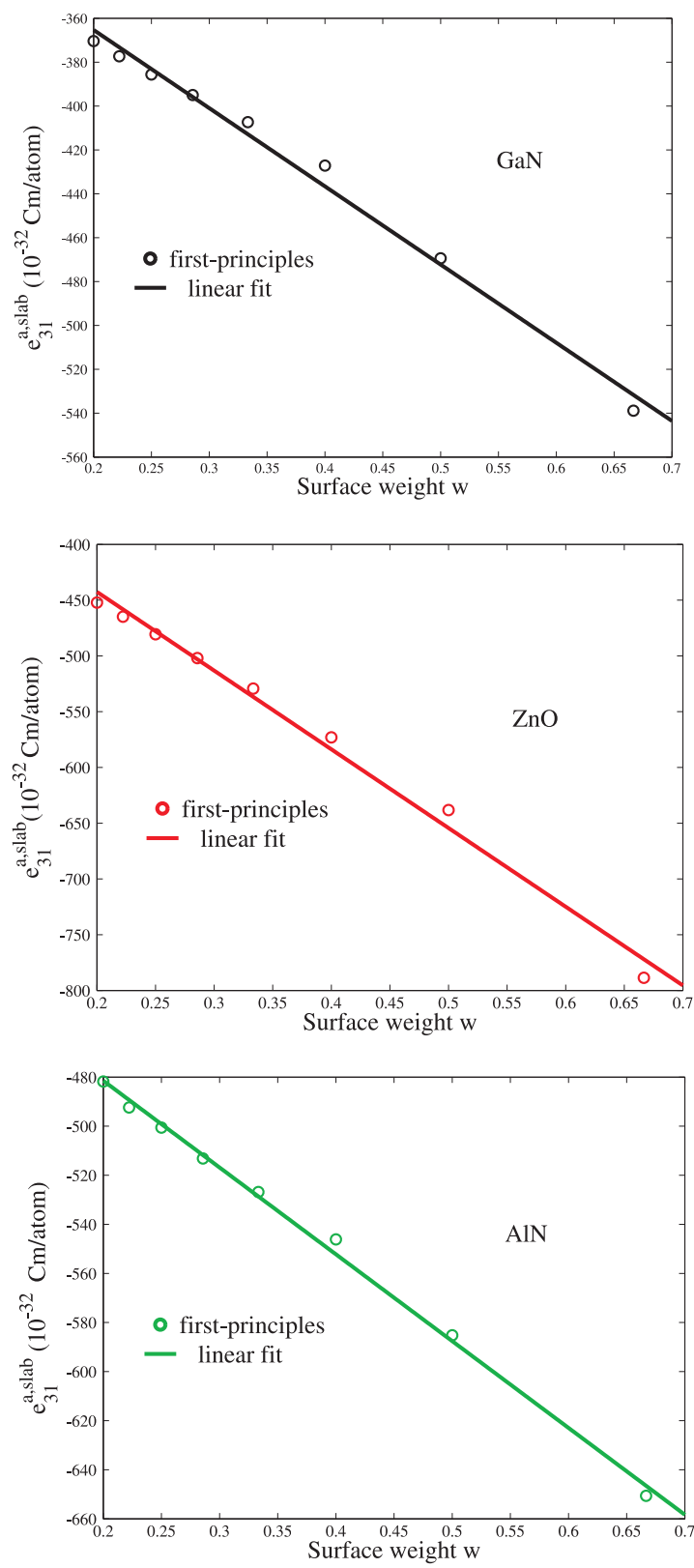

FIG. 4: Plots of $e_{31}^{a, s l a b}$ (in $10^{-32} \mathrm{Cm} /$ atom) as a function of surface weight $w$, used for computing the surface piezoelectric parameter $e_{31}^{s}$ for GaN, $\mathrm{ZnO}$ and $\mathrm{AlN}$

The surface parameters obtained in this way from slab calculations, are then used to model the nanowire parameters. Clearly, for small nanowires, the surface structure can be different from slab affecting the wire properties. However, for nanowires of bigger diameters which are the objects of present continuum model, the difference will show up only at nanowire corners, which represent a small portion of total atoms and can be thus safely neglected. The good agreement between $a b$ initio results and continuum model, as described in next sections, is
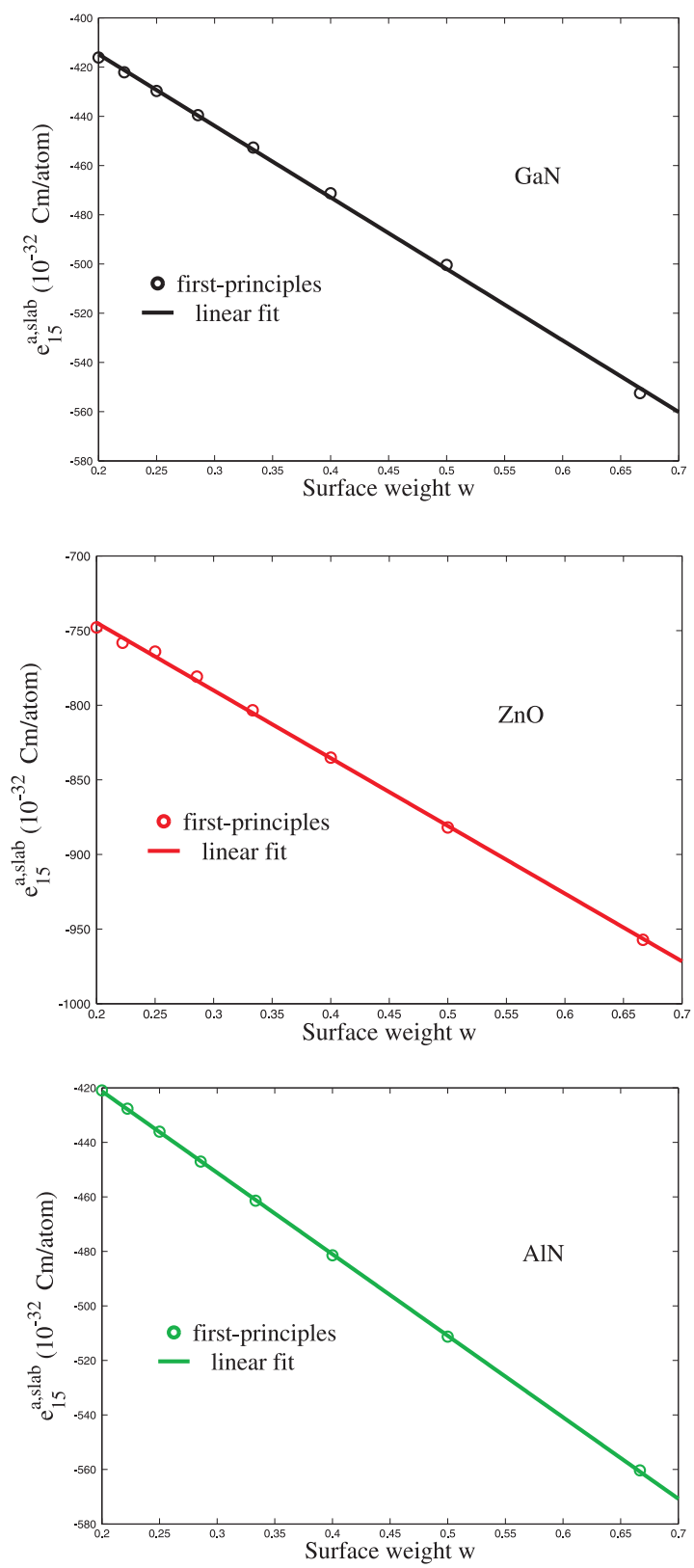

FIG. 5: Plots of $e_{15}^{a, s l a b}$ (in $10^{-32} \mathrm{Cm} /$ atom) as a function of surface weight $w$, used for computing the surface piezoelectric parameter $e_{15}^{s}$ for $\mathrm{GaN}, \mathrm{ZnO}$ and $\mathrm{AlN}$.

an indirect proof of the validity of such an assumption. Still, the structure of surface and the corners of nanowire may certainly be of interest and we plan to publish a detailed study elsewhere. Briefly, we found that while the outmost surface atoms of metal are diving into the bulk, the corner atoms are more relaxed and less shifted. ${ }^{29}$ To give a brief example: for $\mathrm{ZnO}$ surface and using PWGGA functional, the $\mathrm{Zn}$ atoms go in by about $0.32 \AA$ in slabs (compared to experimental values of 0.45 (LEED), 0.06 (GIXD) and 0.37 (HRTEM)), ${ }^{42}$ the central Zn atom on 
TABLE III: Surface elastic parameters (in N/m).

\begin{tabular}{ccccccc}
\hline \hline Method & $C_{11}^{s}$ & $C_{33}^{s}$ & $C_{13}^{s}$ & $C_{55}^{s}$ & $\tau_{1}^{s}$ & $\tau_{3}^{s}$ \\
\hline & & & & & & \\
$G a N$ & & & & & & \\
& & & & & & \\
PWGGA & 99.0 & 78.7 & 18.2 & 33.8 & -1.59 & -1.00 \\
PBESOL & 99.6 & 79.9 & 18.3 & 33.8 & -1.82 & -1.07 \\
PBE0 & 107.3 & 86.5 & 21.4 & 37.2 & -1.97 & -1.18 \\
\hline & & & & & & \\
$Z n O$ & & & & & & \\
PWGGA & 44.2 & 35.0 & 14.2 & 11.7 & -1.80 & -1.30 \\
PBESOL & 42.3 & 32.8 & 13.4 & 10.0 & -1.92 & -1.25 \\
PBE0 & 49.1 & 34.9 & 15.1 & 13.7 & -2.13 & -1.36 \\
\hline & & & & & & \\
AlN & & & & & & \\
PWGGA & 101.7 & 68.0 & 21.2 & 39.9 & -2.20 & -0.78 \\
PBESOL & 99.2 & 64.6 & 21.0 & 39.2 & -2.33 & -0.71 \\
PBE0 & 109.6 & 72.7 & 23.0 & 43.4 & -2.49 & -0.87 \\
B3LYP & 114.1 & 78.0 & 22.3 & 44.3 & -2.33 & -0.87 \\
& & & & & & \\
\hline \hline
\end{tabular}

TABLE IV: Surface piezoelectric parameters (in $\mathrm{nC} / \mathrm{m}$ ) of $\mathrm{GaN}, \mathrm{ZnO}$ and AlN obtained by first-principles calculations.

\begin{tabular}{lccc}
\hline \hline Method & $e_{33}^{s}$ & $e_{31}^{s}$ & $e_{15}^{s}$ \\
\hline GaN & & & \\
PWGGA & 0.375 & -0.143 & -0.153 \\
PBESOL & 0.408 & -0.153 & -0.166 \\
PBE0 & 0.402 & -0.174 & -0.173 \\
\hline ZnO & & & \\
PWGGA & 0.451 & -0.216 & -0.253 \\
PBESOL & 0.470 & -0.229 & -0.274 \\
DFT 28 & 0.496 & -0.263 & \\
\hline & & & \\
AlN & & & \\
PWGGA & 0.586 & -0.188 & -0.170 \\
PBESOL & 0.628 & -0.195 & -0.173 \\
PBE0 & 0.597 & -0.199 & -0.177 \\
& & & \\
\hline \hline
\end{tabular}

the surface of $n=6$ wire goes in by $0.31 \AA$. We also must note that some care should be taken when comparing slab and wire surfaces, as the slab is usually calculated at bulk periodicity while in nanowires the bulk core is somewhat relaxed, see $^{31}$.

\section{COMPARISON BETWEEN CONTINUUM MODEL AND FIRST-PRINCIPLES CALCULATIONS}

To compute the effective piezoelectric coefficients, we prescribe a strain along the axis of the nanowire. The problem is solved by the finite element procedure described in section II. The associated bulk and surface polarizations are deduced from Eqs. (7)-(9) and (10).
The effective piezoelectric constant is then computed numerically from (19). In addition, full first-principles calculations are conducted on nanowires with 48, 108, 192, 300, 432 and 588 atoms per unit cell, corresponding to diameter $d=0.6, d=1.3, d=1.9, d=2.5, d=3.2$ and $d=3.9 \mathrm{~nm}$. The effective piezoelectric constant for the nanowires is defined as:

$$
e_{i k}^{e f f}\left(\mathrm{C} / \mathrm{m}^{2}\right)=e_{i k}^{\text {wire }}\left(10^{-32} \mathrm{Cm} / \text { atom }\right) N_{\text {atoms }} / V^{b} .
$$

where $e_{i k}^{w i r e}$ are the per-atom piezoelectric coefficients for a given nanowire, $N_{\text {atoms }}=12 n^{2}$ is the number of atoms in the unit cell corresponding to the periodic lattice defining the nanowire model, and $V^{b}$ is the volume of the nanowire defined as

$$
V^{b}=\frac{3 \sqrt{3}}{8} d^{2} c
$$

where $d$ is the nanowire diameter, given by $d=2(n-1) a$. The values of the effective coefficient $e_{33}^{e f f}$ of nanowires of different diameters for $\mathrm{GaN}, \mathrm{ZnO}$ and $\mathrm{AlN}$ are reported in Tables V, VI and VII, respectively. Increased piezoelectric effect is observed for nanowires below 2-3 nm, which confirms the results obtained in. ${ }^{10}$ We note, however, that a direct comparison with other theoretical results is not easy as it relies on the same definition of nanowire diameter and thus its volume, which is often not the case. Here we remind that our definition, $d=2(n-1) a$, corresponds to the volume of internal core part of nanowire $\left(12(n-1)^{2}\right.$ atoms) assuming bulk density. This definition is required for consistency with continuum model that considers surface as having zero width but with nonzero elastic and piezoelectric parameters, such as only core atoms contribute to volume. The diameter defined this way is somewhat smaller than 'true' nanowire diameter as surface atoms have nonzero size. This 'true' diameter is however quite difficult to define correctly, because it is unclear what is the atom 'size', and as the electronic density is not uniform. For example, anion oxygen or nitrogen atoms which are less shifted when surface is relaxed, have somewhat bigger size compared to their metal cation counterparts. So, taking diameter as the largest in-plane distance between nuclei is also not quite correct as electrons give important contribution to nanowire structure. In fact, the correct way to compare would be to use the per-atom properties (which though are not defined within continuum model) or, even better, to model the results of real experiments made with nanowires.

In fact, the effective diameter which is somewhat smaller compared to 'true' one (or due to diameter relaxation as concluded in Ref. $)^{10}$ is the main responsible for the fast growth of effective nanowire properties for small d. It also explains why for $\mathrm{ZnO}$ nanowires $e_{33}^{\text {eff }}$ grows for small nanowires while the $e_{33}$ parameter of surface is nearly the same (or even smaller) than that of bulk. We must note however that the surface $e_{31}$ parameter contributes as well to the value of $e_{33}^{e f f}$ for nanowires such as per-atom values do grow (slowly) for small $d$, even for $\mathrm{ZnO}$. 
TABLE V: Effective first-principles piezoelectric parameter $e_{33}^{\text {eff }}$ (in $C / \mathrm{m}^{2}$ ) of $\mathrm{GaN}$ nanowire for different diameters $d$ (in $\mathrm{nm}$ ). Bulk value from $^{10}$ is a bulk parameter which is somewhat smaller than limiting $e_{33}^{e f f}$ value that also includes contribution from bulk $e_{31}$.

\begin{tabular}{lccccccc}
\hline \hline $\mathrm{n}$ & 2 & 3 & 4 & 5 & 6 & 7 & $\infty$ \\
$\mathrm{N}$ & 48 & 108 & 192 & 300 & 432 & 588 & \\
\hline
\end{tabular}

\begin{tabular}{|c|c|c|c|c|c|c|c|}
\hline $\begin{array}{l}P B E S O L \\
\mathrm{~d}\end{array}$ & 0.634 & 1.268 & 1.902 & 2.536 & 3.171 & & Bulk \\
\hline$e_{33}^{e f f}$ & 5.909 & 3.067 & 2.309 & 1.971 & 1.778 & & 1.12 \\
\hline \multicolumn{8}{|l|}{$P B E 0$} \\
\hline d & 0.636 & 1.271 & 1.907 & & & & Bulk \\
\hline$e_{33}^{e f f}$ & 6.003 & 3.127 & 2.358 & & & & 1.15 \\
\hline \multicolumn{7}{|l|}{$P W G G A$} & Bulk \\
\hline$e_{33}^{e f f}$ & 5.299 & 2.699 & 2.025 & 1.715 & 1.541 & 1.428 & 0.94 \\
\hline
\end{tabular}

Reference. $^{10}$

\begin{tabular}{lll}
$\mathrm{d}$ & 1.2 & Bulk \\
\hline
\end{tabular}

$e_{33}$

7.6

0.554

TABLE VI: Effective first-principles piezoelectric parameter $e_{33}^{e f f}$ (in $C / m^{2}$ ) of $\mathrm{ZnO}$ nanowire, with different diameters $d$ (in $\mathrm{nm}$ ). See also caption of Table.V

\begin{tabular}{lccccccc}
\hline \hline $\mathrm{n}$ & 2 & 3 & 4 & 5 & 6 & 7 & $\infty$ \\
$\mathrm{N}$ & 48 & 108 & 192 & 300 & 432 & 588 & \\
\hline
\end{tabular}

\begin{tabular}{llllllll} 
PBESOL & & & & & & & \\
$\mathrm{d}$ & 0.647 & 1.295 & 1.942 & 2.589 & 3.237 & 3.884 & Bulk \\
$e_{33}^{\text {eff }}$ & 7.995 & 4.376 & 3.422 & 2.992 & 2.749 & 2.593 & 1.88 \\
\hline PWGGA & & & & & & & \\
$\mathrm{d}$ & 0.656 & 1.311 & 1.966 & 2.622 & 3.277 & 3.933 & Bulk \\
$e_{33}^{\text {eff }}$ & 7.324 & 3.956 & 3.125 & 2.817 & 2.495 & 2.357 & 1.70 \\
\hline
\end{tabular}

Reference. $^{21}$

\begin{tabular}{lccccc}
$\mathrm{d}$ & 0.932 & 1.561 & 2.197 & 2.833 & Bulk \\
$e_{33}$ & 6.52 & 3.75 & 3.13 & 2.72 & 1.29 \\
Reference. $^{10}$ & & & & & \\
d & 1.2 & & & & Bulk \\
$e_{33}$ & 18.1 & & & & 1.18 \\
\hline
\end{tabular}

TABLE VII: Effective first-principles piezoelectric parameter $e_{33}^{\text {eff }}$ (in $C / \mathrm{m}^{2}$ ) of AlN nanowire, with different diameters $d$ (in $\mathrm{nm}$ ).

\begin{tabular}{lccccccc}
\hline \hline $\mathrm{n}$ & 2 & 3 & 4 & 5 & 6 & 7 & $\infty$ \\
$\mathrm{N}$ & 48 & 108 & 192 & 300 & 432 & 588 & \\
\hline
\end{tabular}

\begin{tabular}{lccccccc} 
PBESOL & & & & & & & \\
$\mathrm{d}$ & 0.622 & 1.247 & 1.867 & 2.489 & 3.115 & & Bulk \\
$e_{33}^{\text {eff }}$ & 9.402 & 5.048 & 3.870 & 3.337 & 3.032 & 1.95 \\
\hline PBE0 & & & & & & & \\
$\mathrm{d}$ & 0.621 & 1.242 & 1.863 & & & & Bulk \\
$e_{33}^{\text {eff }}$ & 9.145 & 4.873 & 3.733 & & & & 1.86 \\
\hline$P W G G A$ & & & & & & & \\
$\mathrm{~d}$ & 0.624 & 1.249 & 1.873 & 2.497 & 3.121 & 3.746 & Bulk \\
$e_{33}^{\text {eff }}$ & 8.687 & 4.620 & 3.534 & 3.029 & 2.753 & 2.571 & 1.73 \\
\hline \hline
\end{tabular}

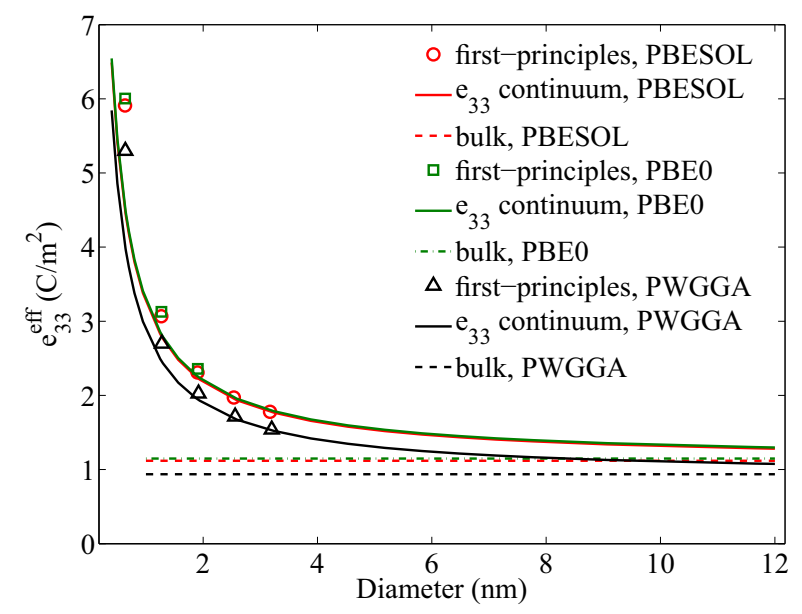

FIG. 6: Effective piezoelectric coefficients $e_{33}^{\text {eff }}$ of GaN nanowire as a function of the diameter; comparison between continuum FEM model and first-principles calculations using PBESOL, PBE0, PWGGA functionals.

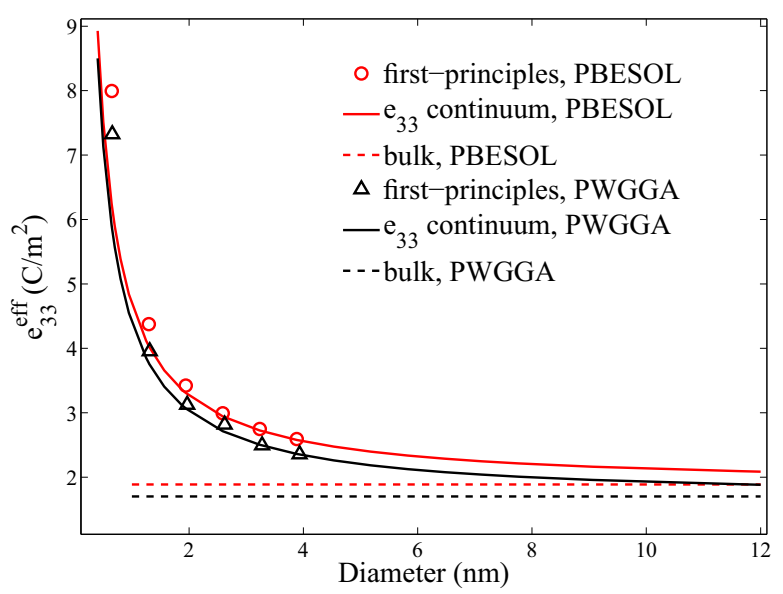

FIG. 7: Effective piezoelectric coefficients $e_{33}^{\text {eff }}$ of $\mathrm{ZnO}$ nanowire as a function of the diameter; comparison between continuum FEM model and first-principles calculations using PBESOL, PBE0, PWGGA functionals.

Plots of the results comparing the values of the effective piezoelectric coefficients $e_{33}^{\text {eff }}$ obtained by the full first-principles and the continuum model are presented in figures 6, 7 and 8 for GaN, $\mathrm{ZnO}$ and AlN, respectively. For each DFT calculation, PWGGA, PBESOL and PBE0 functionals have been used. We can notice a very good agreement between continuous and first-principles results for all materials.

The continuum model allows for computing the nonuniform strain, stress and polarization fields in the nanowire submitted to an arbitrary load, and for arbitrary sizes of nanowires. In figure 9 , we show the polarization field and the distorted surface of a GaN nanowire in absence of load and without electrical field for diam- 


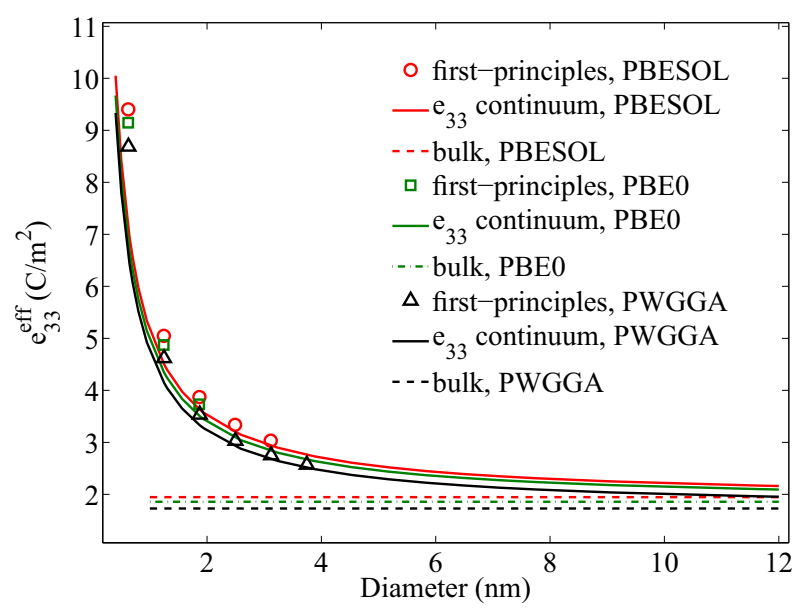

FIG. 8: Effective piezoelectric coefficients $e_{33}^{\text {eff }}$ of AlN nanowire as a function of the diameter; comparison between continuum FEM model and first-principles calculations using PBESOL,PBE, PWGGA functionals.

eters $d=1 \mathrm{~nm}, 3 \mathrm{~nm}$ and $100 \mathrm{~nm}$. We can observe the size effects on both strain and polarization field: for very small nanowires, the residual stress induces a deformation of the surface, while this effect vanishes for larger nanowires. We can also clearly observe a dependence of the local polarization fields on the size of the nanowire.

\section{CONCLUSIONS}

We have proposed a methodology for computation of

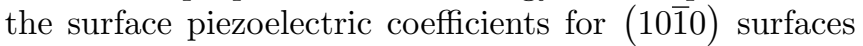
and provide the numerical values for wurtzite AlN, GaN and $\mathrm{ZnO}$ nanowires. The coefficients can be used within a continuum piezoelectric model extended with a surface energy. We have solved the equations of the continuum model with a finite element technique and compared the results with full first-principles calculations of nanowires with diameters ranging from $0.6 \mathrm{~nm}$ to $3.9 \mathrm{~nm}$. A good agreement is found between the continuum and first-principles results for the effective piezoelectric coefficient along the axial direction of the nanowire. For all materials, an increase of the effective piezoelectric coefficients is found when the diameter of the nanowire decreases.
* Electronic address: julien.yvonnet@univ-paris-est.fr

1 Z.L. Wang, Appl. Phys. A 88, 7-15 (2007)

2 Z.L. Wang, X. Wang, J. Song, J. Liu and Y. Gao, IEEE CS. 7, 49-55 (2008).

3 Z.L. Wang, Mater. Sci. Eng. R-Rep. 64, 33-71 (2009)

4 J. Song, J. Zhou, and Z. L. Wang, Nano Lett. 6(8), 1656$1662(2006)$.

${ }^{5}$ X. Xu, A. Potié, R. Songmuang, J. Lee B. Bercu, T. Baron, B. Salem and L. Montes, Nanotech., 22(10), 105704, 2011.

${ }^{6}$ P. X. Gao, J. Song, J. Liu, Z. L. Wang, Adv. Mater 19, 67-72 (2006)

7 J. A. Christman, R. R. Woolcott, A. I. Kingon, and R. J. Nemanicha, Appl. Phys. Lett. 73(26) (1998).

8 M. Minary-Jolandan, R. A. Bernal, I. Kuljanishvili, V. Parpoil, and H. D. Espinosa, Nano Lett., 12(2), 970-976 (2011).

9 H. D. Espinosa, R. A. Bernal, M. M-Jolandan, Adv. Mater. 24, 3289-3431 (2012)

10 R. Agrawal and H. D. Espinosa, Nano Lett. 11(2), 786-790, (2011).

11 Y. Qi and M. C. McAlpine, Energy Environ. Sci, 3, 12751285 (2010).

12 C. Sun, J. Shi, and X. Wang, J. Appl. Phys 108, 034309 (2010)

13 Z. L. Wang, and J. Song, Science 312, 246 (2006)

14 Y. Hu, Y. Zhang, C. Xu, L. Lin, R.L. Snyder and Z. L. Wang, Nano Lett. 11 (6), 2572-2577 (2011).

15 M. Alexe, S. Senz, M. A. Schubert, D. Hesse, and U. Gsele, Adv. Mater, 4021-4026 (2008).

16 Y. Gao, Z. L. Wang, Nano Lett. 7(8), 2499-2505 (2007).

17 M. Lee, J. Bae, J. Lee, C-S Lee, S. Hong and Z. L. Wang, Energy Environ. Sci., 4, 3359-3363 (2011).
18 Y. Li, J. Xiang, F. Qian, S. Gradecak,Y. Wu, H. Yan, D. A. Blom, and C. M. Lieber, Nano Lett. 6 (7), pp 1468-1473 (2006).

19 M. Zhao, Y. Xia, X. Liu, Z. Tan, B. Huang, C. Song, L. Mei, J. Phys. Chem. B. 110, 8764-8768 (2006).

20 M. Rosini, R. Magri, ACS Nano. 26, 6021-31 (2010).

${ }^{21}$ H. J. Xiang, J. Yang, J. G. Hou, and Q. Zhu, Appl. Phys. Lett., 22(89), p. 223111, (2006).

${ }^{22}$ M. Catti, Y. Noel, and R. Dovesi, Phys. Chem. Solids, 64, 2183 (2003).

23 N. A. Hill, U. Waghmare, Phys. Rev. B 62, 8802-8810 (2000).

24 Y. Noel, M. Llunell, R. Orlando, P. Darco, and R. Dovesi, Phys. Rev. B 66, 214107 (2002).

${ }^{25}$ K. Shimada, T. Sota, K. Suzuki, J. Appl. Phys. 84, 4951 (1998).

${ }^{26}$ F. Bernardini, V. Fiorentini, D. Vanderbilt, Phys. Rev. B. 56, R10024-R10027 (1997).

27 C. Li, X. Guo and H. Gao, Appl. Phys. Lett. 90, 0033108 (2007).

28 S. Dai, M. Gharbi, P. Sharma, and H. S. Park, J. Appl. Phys. 110, 104305 (2011).

29 J. Yvonnet, A. Mitrushchenkov, G. Chambaud and Q.-C. He, Comput. Meth. Appl. Mech. eng. 200, 614-645 (2011).

30 J. Yvonnet, A. Mitrushchenkov, G. Chambaud, Q.-C. He and S.-T. Gu, J. Appl. Phys. 111, 124305 (2012).

31 A. Mitrushchenkov, G. Chambaud, J. Yvonnet and Q.-C. He, Nanotech. 21, 255702 (2010).

32 L. C. Lew Yan Voon and M. Willatzen, J. Appl. Phys. 109, 031101 (2011).

33 R. D. King-Smith, D. Vanderbilt, Phys. Rev. B. 47, 1651, (1993). 


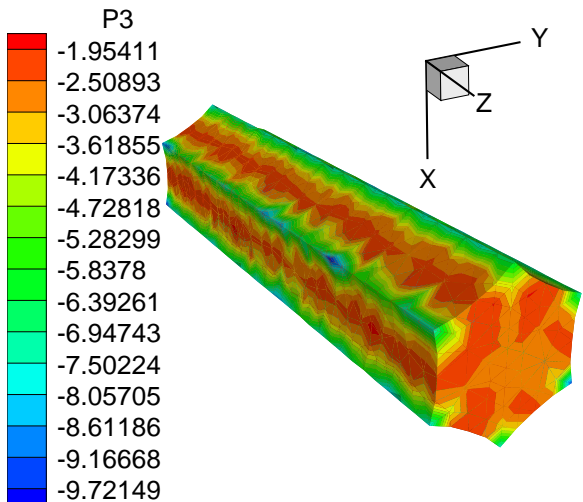

(a)

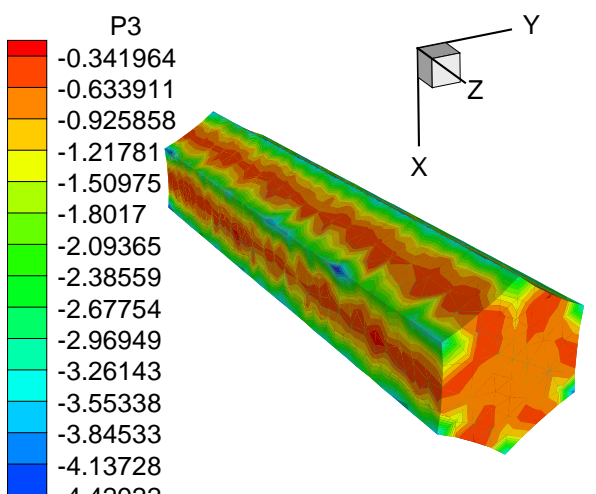

(b)

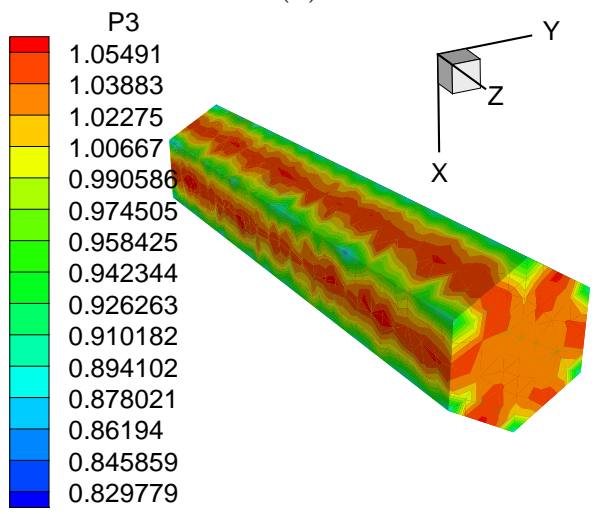

(c)

FIG. 9: Magnified strains and $P_{3}\left(10^{-21} \times C / \mathrm{m}^{2}\right)$ polarization field in GaN nanowire under free relaxation with the continuum FEM model and different diameters: (a) $d=1 \mathrm{~nm}$; (b) $d=3 \mathrm{~nm}$; (c) $d=100 \mathrm{~nm}$. We can note the size effects with the variation of the diameter.
34 D. Vanderbilt, R. D. King-Smith, Phys. Rev. B. 48, 4442, (1993).

35 D. Vanderbilt, J. Phys. Chem. Solids. 61, 147-151, (2000).

36 J.E. Jaffe, N.M. Harrison and A.C. Hess, Phys. Rev. B 49, 11153 (1994).

37 B. Montanari, B. Civalleri, C. M. Zicovich-Wilson, R. Dovesi, Quantum. Chem, 106, 1703 (2006).

38 R. Pandey, J. E. Jaffe, N. M. Harrison, Journal of Physics and Chemistry of Solids, 55, 1357 (1994).

39 R. Pandey, M. Causa, N. M. Harrison, M. Seel, Journal of Physics - Condensed Matter, 8, 3993 (1996).

40 A. Lichanot, M. Chaillet, C. Larrieu, R. Dovesi, C. Pisani, Chem. Phys, 164, 383 (1992).

41 N. Nakamura, H. Ogi, and M. Hirao, Appl. Phys. 111, 013509 (2012)

42 N. L. Marana, V. M. Longo, E. Longo and J. B. L. Martins, J. Phys. Chem. A 112, 89588963 (2011).

43 K. Yoshio, A. Onodera, H. Satoh, N. Sakagami, H. Yamashita, Ferroelectrics 264, 133 (2001).

${ }^{44}$ G. Carlotti, D. Fioretto, G. Socino and E. Verona, J. Phys.: Condens. Matter 7, 9147 (1995).

45 W.F. Perger, J. Criswell, B. Civalleri, R. Dovesi, Comp. Phys. Comm. 180, 1753(2009).

${ }^{46}$ L. E. McNeil, M. Grimsditch, R. H. French, J. Am. Ceram. Soc. 76, 1132 (1993).

47 E. Ruiz, S. Alvarez and P. Alemany, Phys. Rev. B 49, 7115 (1994).

48 H. Iwanaga, A. Kunishige and S. Takeuchi, J. Mater. Sci. 35, 2451 (2000).

49 A. D. Bykhovski, B. L. Gelmont, and M. S. Shur, J. Appl. Phys. 81, 6332 (1997) 